\title{
РЕДУКТИВНЫЕ ОДНОРОДНЫЕ ПРОСТРАНСТВА И НЕАССОЦИАТИВНЫЕ АЛГЕБРЫ
}

\section{§ 1. Предварительные сведения}

Пусть $G-$ связная группа Ли с алгеброй Ли g и $H-$ замкнутая подгруппа в $G$ с подалгеброй Ли h⿱㇒冋. Однородное пространство $M=G / H$ называется редуктивным $\left[{ }^{1,2}\right]$, если в g существует такое подпространство $\mathrm{m}$, называемое редуктивным дополнением или оснащением, что $\mathfrak{g}=\mathfrak{h}+\mathrm{m}$ (прямая сумма) и $(A d H) \mathrm{m} \subset \mathrm{m}$. Локально (в терминах алгебр Ли) второе условие означает $[\mathfrak{h}, \mathrm{m}] \subset \mathrm{m}$, и тогда соответствующая пара $(\mathfrak{g}, \mathfrak{h})$ называется редуктивной парой. Известно, что $G / H$ является редуктивным, например, всегда, когда $H$ компактна нли полупроста ([ $\left.\left.{ }^{1}\right]\right)$. Следуя А. Сэйглу $\left[^{3}\right]$, фиксированное редуктивное дополнение $m$ можно наделить структурой неассоциативной антикоммутативной алгебры, полагая для $X, Y \in m$ произведение $X \cdot Y=[X, Y]_{\mathfrak{m}}$, где $[X, Y]_{\mathrm{m}}$ - проекция скобки $[X, Y]$ на подпространство $\mathrm{m}$ (аналогично: $[X, Y]_{\mathfrak{h}}-$ проекция скобки $[X, Y]$ на $\left.\mathfrak{h}\right)$. Строение этой алгебры, которую мы будем обозначать через $(\mathrm{m}, \cdot)$ или просто через $\mathrm{m}$, тесно связано с геометрией соответствующего редуктивного пространства $G / H\left[{ }^{3,4}\right]$. Однако алгебра m не является единственной неассоциативной алгеброй, связанной с $G / H$. В силу результата K. Номидзу $\left[{ }^{2}\right]$, на редуктивном пространстве $G / H$ с фиксированным разложением $\mathfrak{g}=\mathfrak{h}+\mathfrak{m}$ существует биективное соответствие между множеством $G$-инвариантных связностей на $G / H$ и множеством билинейных функций $\alpha: \mathrm{m} \times \mathrm{m} \rightarrow \mathrm{m}$, которые $\operatorname{Ad}(H)$-инвариантны, т. е. с каждой $G$-инвариантной связностью на $G / H$ связывается некоторая неассоциативная алгебра $(\mathrm{m}, \alpha)$, для которой $\operatorname{Ad}(H) \subset \operatorname{Aut}(\mathrm{m}, \alpha)$. Из всех инвариантных связностей на $M=G / H$ выделяется естественная связность без кручения $\left[{ }^{2}\right]$, для которой $\alpha(X, Y)=1 / 2 X \cdot Y$. Алгеброй, соответствующей этой связности, является, очевидно, алгебра m.

В дальнейшем метрику с естественной связностью без кручения будем обозначать буквой $B$. Однородное пространство $G / H$ с такой метрикой называется естественно-редуктивным. Примером такого пространства является, в частности, однородное пространство, локально определяемое парой Киллннга $(\mathfrak{g}, \mathfrak{h})\left[{ }^{5}\right]$.

Линейную группу голономии инвариантной связности $\alpha$ на однородном пространстве $G / H$ будем обозначать через $\mathrm{Hol}(\alpha)$ и алгебру голономии через hol $(\alpha)$. Если рассматривать только односвязные $G / H$, то $\mathrm{Hol}(\alpha)$ всегда связна, потому $\mathrm{Hol}(\alpha)=\operatorname{exphol}(\alpha)$ и действие $\mathrm{Hol}(\alpha)$ на m равносильно действию hol $(\alpha)$ на m. В данной работе все редуктивные однородные пространства предполагаются односвязными. 
О п р еделе н и е 1. Односвязное однородное пространство $\mathrm{G} / \mathrm{H}$ называется голономно неприводимым относительно связности а, если hol $(\alpha)$ действует неприводимо на m.

Известная теорема Ж. де Рама $\left[{ }^{6}\right]$, согласно которой всякое полное односвязное риманово многообразие изометрично прямому произведению односвязных голономно неприводимых римановых многообразий, стимулирует исследование голономно неприводимых пространств. Строение алгебры м связано с голономной неприводимостыо редуктивного $G / H$ следующим результатом.

T е о рем а 1. (Сэйгл $\left.\left[{ }^{3}\right]\right)$. Пусть $G / H-$ односвязное редуктивное однородное пространство с разложением $\mathrm{g}=\mathrm{h}+\mathrm{m}$. Если $\mathrm{m}^{2}=$ $=\mathrm{m} \cdot \mathrm{m} \neq 0$ u $\mathrm{G} / \mathrm{H}$ голономно неприводимо относительно естественной связности без кручения, то алгебра щ проста. Обратно: если $G / H$ является псевдоримановым пространством и п проста, то $G / H$ голономно неприводимо.

В случае риманова однородного пространства имеет место более сильное утверждение.

T е о р е а 2. (Костант $\left.\left[{ }^{7}\right]\right)$. Пусть $G / H-$ односвязное риманово естественно-редуктивное однородное пространство. Оно голономно неприводимо относительно естественной связности без кручения тогда и только тогда, когда $G$ проста.

В настоящей работе мы продолжаем сравнительное изучение алгебр $(m, \alpha)$ и $m$, начатое Б. Костантом $\left[{ }^{8}\right]$, и выясняем взаимосвязь строения этих алгебр и соответствующего редуктивного пространства $G / H$, опираясь на результаты, полученные А. Сэйглом $\left[{ }^{4}\right]$. Основной результат: односвязное однородное естественно-редуктивное пространство при определенных условиях, накладываемых на инфинитезимальную структуру, разлагается в прямое произведение свонх вполне геодезических подмногообразий или же голономно неприводимо (относительно естественной связности без кручения).

\section{§ 2. Алгебры псевдоримановых связностей}

Пр едл о жени е 1 . Пусть $G / H$ - односвязное редуктивное однородное пространство с разложением $\mathrm{g}=\mathrm{h}+\mathrm{m} u \alpha-$ инвариантная псевдориманова связность на G/H. Тогда простота алгебры м влечет простоту алгебры $(11, \alpha)$.

Д ок а з а т л в с в о. Рассматриваемая связность $\alpha$ является связностью без кручения и потому

$$
\operatorname{Tor}(X, Y)=\alpha(X, Y)-\alpha(Y, X)-X \cdot Y=0 .
$$

Отсюда сразу следует, что $(\mathrm{m}, \alpha)$ - алгебра с ненулевым умножением. Действительно, если имеет место обратное, то из соотношения (1) получаем $X \cdot Y=0$ для любых $X, Y \in \mathrm{m}$, но тогда $\mathrm{m}^{2}=0$, что противоречит простоте щ. Если теперь $(m, \alpha)$ содержит двусторонний идеал $п$, то опять же из (1) получаем противоречие: $n$ является идеалом и в алгебре $\mathrm{m}$.

Пр едложен и е 2. Пусть $G / H-$ односвязное однородное пространство с компактной $G$ и $\mathrm{m}$ - ортогональное дополнение $\kappa \mathfrak{h}$ относительно положительно определенной квадратичной формы $B$ на g. Тогда простота $G$ влечет простоту алгебры $(\mathrm{m}, \alpha)$, где $\alpha-$ произвольная инвариантная псевдориманова связность на $\mathrm{G} / \mathrm{H}$.

Д ока з а тельство. Пусть алгебра $(m, \alpha)$ не проста, тогда не проста и алгебра m (предложение 1 ) и потому $G / H$ голономно приво- 
димо относительно естественной связности без кручения (теорема 1). Но это противоречит голономной неприводимости риманова редуктивного однородного пространства $G / H$ с простой $G$ относительно естественной связности без кручения (теорема 2).

Предло жение 3 . Пусть $G / H$ - односвязное редуктивное несимметрическое пространство с инвариантной псевдоримановой связностью $\alpha$. Если $G / H$ голономно неприводимо относительно $\alpha$, то алгебра $(\mathrm{m}, \alpha)$ - проста.

Доказательство. Если $\alpha(X, Y)=0$ для любых $X, Y \in \mathrm{m}$, то $0=\operatorname{Tor}(X, Y)=-X \cdot Y$, т. е. $G / H$ оказывается симметрическим, что противоречит условию. Пусть $(\mathrm{m}, \alpha)$ содержит собственный идеал. Тогда, согласно $\left[{ }^{3}\right]$, она содержит собственный идеал $\mathrm{n}$, инвариантный относительно hol $(\alpha)$, что противоречит голономной неприводимости $G / H$.

Пусть $(\mathfrak{g}, \mathfrak{h})$ - редуктивная пара с разложением $\mathfrak{g}=\mathfrak{h}+\mathfrak{m}$. Следуя А. М. Васильеву $\left[{ }^{9}\right]$, назовем подалгебру $\mathfrak{g}^{\prime} \subset g$ нормальной, если

$$
\mathfrak{g}^{\prime}=\mathfrak{g}^{\prime} \cap \mathfrak{h}+\mathfrak{g}^{\prime} \cap \mathrm{m} \text {. }
$$

Такая подалгебра определяет в соответствующем пространстве $G / H$ вполне геодезическое подмногообразие относительно связности, определяемой оснащением м. Оснащение т называется «проникающим в g» $\left(\left[{ }^{7}\right]\right)$, если в редуктивном разложении $\mathfrak{g}=\mathfrak{h}-\mathrm{i}$ подалгебра $\mathfrak{h}=$ $=[\mathrm{m}, \mathrm{m}]_{\mathfrak{h}}$. Таким оно является, в частности, в случае простой алгебры h $\left(\left[{ }^{10}\right]\right)$. Следующая теорема дополняет результат Б. Костанта ([ $\left.{ }^{7}\right]$, теорема 5) о голономной приводимости римановых однородных пространств.

T е о р е а 3. Пусть $G / H$ - односвязное редуктивное несимметрическое однородное пространство с разложением $\mathrm{g}=\mathfrak{h}+\mathrm{m} u \mathrm{~m}$ - «проникающее в g» оснащцение. Если $G / H$ голономно неприводимо относительно естественной связности без кручения, то g не содержит собственных нормальных идеалов.

Доказ ательство. В силу теоремы 1 алгебра $m$ проста и пусть g содержит собственный нормальный идеал $\mathfrak{g}^{\prime}=\mathfrak{g}^{\prime} \cap \mathfrak{h}+\mathfrak{g}^{\prime} \cap \mathrm{m}$. Предполагая действие группы эффективным, можем считать $\mathrm{g}^{\prime} \cap \mathrm{m} \neq 0$, так как в противном случае подалгебра $\mathfrak{h}$ будет содержать идеал $\mathfrak{g}^{\prime} \cap \mathfrak{h}$, являющийся идеалом и в $\mathfrak{g}$. По условию $\left[\mathfrak{g}^{\prime}, \mathrm{m}\right]=\left[\mathfrak{g}^{\prime} \cap \mathfrak{h}+\mathfrak{g}^{\prime} \cap \mathrm{m}, \mathrm{m}\right] \subset$ $\subset \mathfrak{g}^{\prime} \cap \mathfrak{h}+\mathfrak{g}^{\prime} \cap \mathrm{m}, \quad$ и потому $\left[\mathfrak{g}^{\prime} \cap \mathfrak{h}, \mathrm{m}\right]+\left[\mathfrak{g}^{\prime} \cap \mathrm{m}, \mathrm{m}\right]_{\mathrm{m}} \subset \mathfrak{g}^{\prime} \cap \mathrm{m}$. Но $\left[\mathfrak{g}^{\prime} \cap \mathfrak{h}, \mathrm{m}\right] \subset\left(\mathfrak{g}^{\prime} \cap \mathfrak{h}+\mathfrak{g}^{\prime} \cap \mathrm{m}\right) \cap \mathrm{m}=\mathrm{g}^{\prime} \cap \mathrm{m}, \quad$ следовательно, $\quad\left[\mathfrak{g}^{\prime} \cap \mathrm{m}\right.$, 巨 $\mathrm{m}=\left(\mathfrak{g}^{\prime} \cap \mathrm{m}\right) \cdot \mathrm{m} \subset \mathfrak{g}^{\prime} \cap \mathrm{m}$, т. е. $\mathfrak{g}^{\prime} \cap \mathrm{m}-$ идеал в $\mathrm{m}$. Ввиду простоты $m$ возможно лишь $g^{\prime} \cap \mathrm{m}=\mathrm{m}$, но тогда $\left[\mathrm{g}^{\prime} \cap \mathfrak{b}+\mathrm{m}, \mathrm{m}\right] \subset \mathrm{g}^{\prime} \cap \mathfrak{b}+\mathrm{m}$ и необходимо $[\mathrm{m}, \mathrm{m}]_{\mathfrak{h}} \subset \mathfrak{g}^{\prime} \cap \mathfrak{h}$. По условию $[\mathrm{m}, \mathrm{m}]_{\mathfrak{h}}=\mathfrak{h}$, что влечет $\mathfrak{g}^{\prime} \cap \mathfrak{h}=\mathfrak{h}$. Полученное противоречие доказывает теорему.

\section{§ 3. Об одном классе естественно-редуктивных пространств}

Рассмотрим редуктивные однородные пространства $G / H$, для которых редуктивная структура задается подпространством

$$
\mathrm{m}=\{X \in \mathrm{g} \mid B(X, Y)=0 \quad \forall Y \in \mathfrak{h}\}
$$

и ограничение формы $B$ на подалгебре $\mathfrak{h}$ невырождено. Поскольку в этом случае $B(X \cdot Y, Z)=B(X, Y \cdot Z)$ для любых $X, Y, Z \in \mathrm{m}$, то $G / H$ является естественно-редуктивным пространством $\left[{ }^{6}\right]$. 
О п р еделени е 2. Редуктивное однородное пространство $\mathrm{G} / \mathrm{H}$ c фиксированным разложением $\mathrm{g}=\mathrm{h}+\mathrm{m}$ называется пространством $c$ идеально ненулевым умножением в $\mathrm{m}$, если алгебра m не содержит идеалов с нулевым умножением.

Следующее предложение указывает на существование таких однородных пространств. Для его формулировки отметим, что на алгебре $\mathrm{m}$, равно как и на произвольной конечномерной алгебре, можно определить форму Киллинга $\widetilde{B}(X, Y)$, полагая

$$
\widetilde{B}(X, Y)=\operatorname{tr}(L(X) L(Y)),
$$

где $L(X): \mathrm{m} \rightarrow \mathrm{m}, Y \rightarrow X \cdot Y$ для $X, Y \in \mathrm{m}$.

Пре дл о жен и е 4. Пусть $G$ - полупростая связная группа Ли $и$ $H$ - ее замкнутая подгруппа. Пусть В-форма Киллинга алгебрь $\mathrm{g}$, ограничение $B_{\mathfrak{h}}$ которой невырождено, $и$ m - ортогональное дополнение $\kappa \mathfrak{h}$ относительно B. Если ограничение формы $B$ на m совпадает с формой Киллинга самой алгебры m, то G/H есть естественно-редуктивное пространство с идеально ненулевым умножением в $\mathrm{m}$.

Д ок а 3 а тельст в о. Пусть $\mathrm{n}$ - идеал в ловию $\mathrm{n}^{2}=0$. Выберем базис в $\mathrm{n}$ и дополним его до базиса в $\mathrm{m}$. Тогда для $X \in \mathrm{n}, Y \in \mathrm{m}$ преобразования $L(X)$ и $L(Y)$ примут вид

$$
\left(\begin{array}{ll}
0 & 0 \\
* & 0
\end{array}\right) \text { и }\left(\begin{array}{ll}
* & 0 \\
* & *
\end{array}\right)
$$

соответственно. Следовательно, $\operatorname{tr}(L(X) L(Y))=0$ и потому $\widetilde{B}(\mathrm{n}, \mathrm{m})=$ $=0$. Отсюда следует, что в случае невырожденности формы $\widetilde{B}$ алгебра m не содержит идеалов $\mathrm{n}$, удовлетворяющих условию $\mathrm{n}^{2}=0$. Теперь наше утверждение будет вытекать из невырожденности ограничения формы Киллинга $B$ алгебры g на m.

Прежде чем дать геометрическую характеристику введенных пространств, уточним формулировку известной теоремы Ж. де Рама $\left[{ }^{6}\right]$ на случай естественно-редуктивных римановых однородных пространств.

Т е о рем а 4. Пусть $M=G / H$ - односвязное риманово естественноредуктивное однородное пространство с разложением $\mathrm{g}=\mathfrak{h} f \mathrm{~m} u$ Ф - группа голономии римановой связности. Тогда

1) имеет место единственное с точностью до порядка разложение

$$
\mathrm{m}=\overline{\mathrm{m}}_{0} \oplus \overline{\mathrm{m}}_{1} \oplus \ldots \oplus \overline{\mathrm{m}}_{p}
$$

в прямую сумму взаимно ортогональных Ф-инвариантных подпространств; Ф действует тривиальнона $\overline{\mathrm{m}}_{0}$ и неприводимо на $\overline{\mathrm{m}}_{j}(1 \leqslant j \leqslant p)$;

2) имеет место соответствующее разложение $M=M_{0} \times M_{1} \times \ldots$ $\ldots \times M_{p}$ в прямое произведение римановых пространств таких, что $\overline{\mathrm{m}}_{j}$ является касательным пространством для $M_{j}(0 \leqslant j \leqslant p)$.

Заметим, что в разложении (2) каждое $\overline{\mathrm{m}}_{j}(0 \leqslant j \leqslant p)$ является идеалом в $\mathrm{m}$, так как $\left[\bar{m}_{j}, \bar{m}_{j}\right] \subset \mathfrak{h}+\bar{m}_{j}$ и $\bar{m}_{i} \cdot \bar{m}_{j}=0$ для $i \neq j \quad\left(\left[{ }^{6}\right]\right.$, c. 213). Далее, всякий идеал с нулевым умножением $\bar{m}_{k}$ алгебры m является центральным (действительно, $\bar{m}_{k} \cdot m=\bar{m}_{k} \cdot \bar{m}_{k}=0$ ) и потому алгебра m разложима в прямую сумму своего центра $z$ и подалгебры f, не содержащей идеалов с нулевым умножением, подобно тому, как любая редуктивная алгебра Ли разложима в прямую сумму своего центра и полупростой подалгебры. В разложении (2) можно изменить порядок таким образом, чтобы $z=\bar{m}_{0} \oplus \ldots \oplus \bar{m}_{s}$ и $\mathfrak{f}=\bar{m}_{s+1} \oplus \ldots \oplus \bar{m}_{p}$ 
давали, соответственно, центр и полупростую подалгебру алгебры m. Итак, справедлива

Т еорема 5. Пусть $G / H$ - односвязное риманово естественно-редуктивное однородное пространство с фиксированным разложением $\mathrm{g}=\mathfrak{h}+\mathrm{m}$. Тогда $\mathrm{m}=z \oplus \mathfrak{t}$, где $\mathrm{z} u \mathfrak{f}-$ идеалы $8 \mathrm{~m}$, причем $z \cdot \mathrm{m}=0$. Нмеет место соответствующее разложение

$$
G / H=G_{1} / H \times G_{2} / H,
$$

где $G_{1} / H$ - симметрическое пространство с касательным пространством з, а $\mathrm{G}_{2} / \mathrm{H}$ - несимметрическое пространство с идеально ненулевым умножением в касательном пространстве f.

Из данной теоремы следует, что односвязные римановы естественноредуктивные однородные пространства с идеально ненулевым умножением в фиксированном редуктивном дополнении m образуют естественный класс редуктивных «максимально не симметрических» пространств.

T е о р м а 6. Пусть $G / H$ - односвязное естественно-редуктивное однородное пространство с метрикой $B$ и идеально ненулевым умножением в редуктивном дополнении п. Если $G / H$ голономно приводимо, то m является прямой суммой hol $(B)$-инвариантных простых идеалов

$$
\mathrm{m}=\mathrm{m}_{0} \oplus \mathrm{m}_{1} \oplus \ldots \oplus \mathrm{m}_{r}
$$

причем это разложение ортогонально относительно $B$ и

(а) $\left[\mathfrak{h}, \mathrm{m}_{i}\right] \subset \mathrm{m}_{i}$;

(б) $\left[\mathrm{m}_{i}, \mathrm{~m}_{i}\right] \subset \mathfrak{h}+\mathrm{m}_{i} \quad\left(\mathrm{~m}_{i} \cdot \mathrm{m}_{i} \subset \mathrm{m}_{i}\right)$;

(в) $\left[\mathrm{m}_{i}, \mathrm{~m}_{j}\right]=0, \quad i \neq j ; \quad(i, j=0,1, \ldots, r)$.

Доказательство. В силу теоремы 1 алгебра m содержит собственный идеал. Тогда множество собственных $D(\mathfrak{h})$-инвариантных идеалов алгебры m не пусто. Пусть идеал по минимален в этом множестве, т. е. $m_{0} \neq 0$, и не существует идеалов, строго промежуточных между 0 и $\mathrm{m}_{0}$. Пусть, далее, $\mathrm{m}_{0}^{\prime}$ обозначает ортогональное дополнение $\mathrm{k} \mathrm{m}_{0}$ относительно $B$, т. е. $\mathrm{m}_{0}^{\prime}=\left\{X \in \mathrm{m} \mid B\left(X, \mathrm{~m}_{0}\right)=0\right\}$. В силу равенств

$$
\begin{aligned}
& B\left(\left[\mathrm{~m}_{0}^{\prime}, \mathfrak{h}\right], \mathrm{m}_{0}\right)=B\left(\mathrm{~m}^{\prime}{ }_{0},\left[\mathfrak{h}, \mathrm{m}_{0}\right]\right)=0, \\
& B\left(\mathrm{~m}_{0}^{\prime} \cdot \mathrm{m}, \mathrm{m}_{0}\right)=B\left(\mathrm{~m}_{0}^{\prime}, \mathrm{m} \cdot \mathrm{m}_{0}\right)=0
\end{aligned}
$$

оно также является $D(\mathfrak{h})$-инвариантным идеалом в $m_{0} \cap m_{0}^{\prime}=0$. Если это не так, то минимальность $m_{0}$ влечет включение $\mathrm{m}_{0} \subset \mathrm{m}_{0}^{\prime}$. Әто означает, что $B\left(\mathrm{~m}_{0}, \mathrm{~m}_{0}\right)=0$, откуда $B\left(\mathrm{~m}_{0}^{2}, \mathrm{~m}\right)=$ $=B\left(\mathrm{~m}_{0}, \mathrm{~m}_{0} \cdot \mathrm{m}\right)=0$. Следовательно, $\mathrm{m}_{0}^{2}=0$, вопреки условню. Итак, $\mathrm{m}_{0} \cap \mathrm{m}_{0}^{\prime}=0$, и потому $\mathrm{m}$ является прямой суммой $\mathrm{m}_{0}$ и $\mathrm{m}^{\prime}$. Равенство $B\left(\mathrm{~m}_{0}, \mathrm{~m}_{0}^{\prime}\right)=0$ влечет $0=B\left(\mathrm{~m}_{0}, \mathrm{~m}_{0}^{\prime} \cdot \mathrm{m}\right)=B\left(\mathrm{~m}_{0} \cdot \mathrm{m}_{0}^{\prime}, \mathrm{m}\right)$. Но $B\left(\mathrm{~m}_{0} \cdot \mathrm{m}^{\prime}, \mathfrak{h}\right)=0$, потому $B\left(\mathrm{~m}_{0} \cdot \mathrm{m}^{\prime}{ }_{0}, \mathfrak{g}\right)=0$ и $\mathrm{m}_{0} \cdot \mathrm{m}^{\prime}{ }_{0}=0$. Следовательно, $\left[\mathrm{m}_{0}, \mathrm{~m}_{0}^{\prime}\right] \subset \mathfrak{h}$ и $B\left(\left[\mathrm{~m}_{0}, \mathrm{~m}_{0}^{\prime}\right], \mathfrak{m}\right)=0 . \quad$ Далее, $B\left(\left[\mathrm{~m}_{0}, \mathrm{~m}_{0}^{\prime}\right], \mathfrak{h}\right)=$ $=B\left(\mathrm{~m}_{0},\left[\mathrm{~m}_{0}^{\prime}, \mathfrak{h}\right]\right) \subset B\left(\mathrm{~m}_{0}, \mathrm{~m}_{0}^{\prime}\right)=0$. B итоге $B\left(\left[\mathrm{~m}_{0}, \mathrm{~m}_{0}^{\prime}\right], \mathrm{g}\right)=0$ и $\left[\mathrm{m}_{0}, \mathrm{~m}^{\prime}{ }_{0}\right]=0$.

Из включений $[\mathfrak{h}, \mathfrak{h}] \subset \mathfrak{h},\left[\mathfrak{h}, \mathfrak{m}_{0}\right] \subset \mathrm{m}_{0}, \quad\left[\mathrm{~m}_{0}, \mathrm{~m}_{0}\right] \subset \mathfrak{h}+\mathrm{m}_{0}$ вытекает, что подпространство $\mathfrak{y}_{0}=\mathfrak{h}+\mathrm{m}_{0}$ является подалгеброй Ли в g. Так как $B\left(\mathfrak{h}_{0}, \mathrm{~m}^{\prime}\right)=0$, то $G / H_{0}$ - естественно-редуктивное пространство, где $H_{0}$ - связная подгруппа в $G$, имеющая $\mathfrak{h}_{0}$ своей алгеброй Ли. Однородное пространство $G / H_{0}$ есть пространство с идеалыно ненулевым умножением в $\mathrm{m}_{0}^{\prime}$. Действительно, если $\mathrm{n}-$ такой идеал в $\mathrm{m}_{0}^{\prime}$, что $\mathrm{n}^{2}=0$, то $n$ является идеалом и в $m$, так как $n \cdot m=n \cdot\left(m_{0}+m^{\prime}{ }_{0}\right)=$ $=\mathrm{n} \cdot \mathrm{m}_{0}^{\prime} \subset \mathrm{n}$ (поскольку $\mathrm{n} \cdot \mathrm{m}_{0}=0$ ), что противоречит условию. Теперь, 
если $\mathrm{m}_{0}^{\prime}$ проста, дальнейшее разложение невозможно. Если же $\mathrm{m}_{0}^{\prime}$ не проста, то условия теоремы наследуются пространством $G / H_{0}$, и процесс продолжается до тех пор, пока $\mathrm{m}$ не разложится в прямую сумму простых идеалов, взаимно ортогональных относительно формы $B$. Так как алгебра голономии $\operatorname{hol}(B)$ связности, определяемой формой $B$, порождена всеми отображениями $L(X)\left(\left[{ }^{4}\right]\right)$, то все $\mathrm{m}_{i}$ являются hol $(B)$ инвариантными. Заключения (а) - (в) следуют непосредственно из доказательства.

Покажем, что полученное разложение (3) является аналогом разложения де Рама касательного пространства $T_{0}(M)=m$ односвязного риманова естественно-редуктивного пространства $M$.

Т еорем а 7. Пусть $M=G / H$ - односвязное риманово естественно-редуктивное однородное пространство с идеально ненулевым умножением в касательном пространстве $T_{0}(M)=m$. Тогда разложение (3) алгебры м является разложением де Рама касательного пространства $T_{0}(M)$.

Доказательство. Как было указано выше, в разложении де Рама (2) касательного пространства $T_{0}(M)=m$ каждое $\bar{m}_{j}$ является идеалом в m. Далее, так как риманова связность, определяемая положительно определенной формой $B$ на $\mathrm{m}$, совпадает с канонической связностью без кручения, то алгебра голономии hol $(B)$ этой связности совпадает с алгеброй Ли, определяемой отображениями $L(X): m \rightarrow \mathrm{m}$, $Y \mapsto X \cdot Y\left(\left[^{4}\right]\right)$. Пусть некоторая алгебра $\bar{m}_{j}$ непроста. Так как в предположениях теоремы $\overline{\mathrm{m}}_{j}^{2} \neq 0$, то $\overline{\mathrm{m}}_{j}$ должна содержать собственный идеал $\bar{n}_{j}$. В силу положительной определенности $B$ на m подпространство

$$
\overline{\mathrm{m}}^{\prime}{ }_{j}=\left\{X \in \mathrm{m} \mid B\left(X, \mathrm{~m}_{j}\right)=0\right\}
$$

представляет собой прямое слагаемое в $\mathrm{m}\left(\mathrm{m}=\overline{\mathfrak{m}}_{j} \oplus \overline{\mathrm{m}}_{j}{ }^{\prime}\right)$ и, в силу $\overline{n_{j}} \cdot \bar{m}_{j}^{\prime}=0$, подпространство $\bar{n}_{j}$ является идеалом и в m, т. е. $L(m)-$ приводимым подпространством, что противоречит неприводимости hol $(B)$ на $\bar{m}_{j}$. Следовательно, все идеалы $\bar{m}_{j}$ просты, и теперь утверждение теоремы следует непосредственно из результата о единственности разложения конечномерной неассоциативной алгебры в прямую сумму простых идеалов.

Т еорем а 8. Пусть. $G / H$ - односвязное естественно-редуктивное однородное пространство. Согласно иредположениям теоремы 6,

если $\mathrm{m}=\bigoplus_{i=1}^{r} \mathrm{~m}_{i}-$ разложение (3), то

(1) $\mathrm{g}_{i}=\left[\mathrm{m}_{i}, \mathrm{~m}_{i}\right] \mathfrak{h}+\mathrm{m}_{i}$ являются идеалами в $\mathrm{g}$;

кроме того, если ш «роникает в g», то

(2) $g=g_{0} \oplus g_{1} \oplus \ldots \oplus g_{r}$.

Доказа тельство. Так как каждое $\mathfrak{m}_{i}$ является $D(\mathfrak{h})$-инвариантным, то подпространства $\mathfrak{h}_{i}=\left[\mathrm{m}_{i}, \mathrm{~m}_{i}\right]_{\mathfrak{h}}-$ идеалы в $\mathfrak{h}\left(\left[{ }^{7}\right]\right)$, и потому

$$
\left[\mathfrak{g}_{i}, \mathfrak{h}\right]=\left[\left[\mathrm{m}_{i}, \mathrm{~m}_{i}\right]_{\mathfrak{h}}+\mathrm{m}_{i}, \mathfrak{h}\right] \subset\left[\mathfrak{m}_{i}, \mathrm{~m}_{i}\right]_{\mathfrak{h}}+\mathrm{m}_{i} \text {. }
$$

Далее

$\left[\mathrm{m}_{i}, \mathrm{~m}\right]=\left[\mathrm{m}_{i}, \mathrm{~m}_{0}+\ldots+\mathrm{m}_{r}\right]=\left[\mathrm{m}_{i}, \mathrm{~m}_{i}\right] \subset\left[\mathrm{m}_{i}, \mathrm{~m}_{i}\right]_{\mathfrak{h}}+\mathrm{m}_{i}=\mathrm{g}_{i}$,

и, наконец, 


$$
\begin{gathered}
{\left[\left[\mathrm{m}_{i}, \mathrm{~m}_{i}\right]_{\mathfrak{h}}, \mathrm{m}\right] \subset\left[\left[\mathrm{m}_{i}, \mathrm{~m}_{i}\right], \mathrm{m}\right] \subset\left[\left[\mathrm{m}_{i}, \mathrm{~m}\right], \mathrm{m}_{i}\right]=} \\
=\left[\left[\mathrm{m}_{i}, \mathrm{~m}\right]_{\mathfrak{h}}+\mathrm{m}_{i} \cdot \mathrm{m}, \mathrm{m}_{i}\right] \subset\left[\left[\mathrm{m}_{i}, \mathrm{~m}_{\mathfrak{h}}\right]_{\mathfrak{h}}+\mathrm{m}_{i}, \mathrm{~m}_{i}\right] \subset \\
\subset\left[\mathrm{m}_{i}, \mathrm{~m}_{i}\right]_{\mathfrak{h}}+\mathrm{m}_{i}=\mathfrak{g}_{i} .
\end{gathered}
$$

Тем самым доказано, что $\left[\mathrm{g}_{i}, \mathrm{~m}\right] \subset \mathfrak{g}_{i}$, вследствие чего подпространства $g_{i}$ являются идеалами в g. Далее,

$$
\mathfrak{h}=\left[\mathrm{m}, \mathrm{m}_{\mathfrak{h}}=\left[\sum_{i=0}^{r} \mathrm{~m}_{i}, \sum_{i=0}^{r} \mathrm{~m}_{i}\right]_{\mathfrak{h}}=\sum_{i=0}^{r}\left[\mathrm{~m}_{i}, \mathrm{~m}_{i}\right]_{\mathfrak{h}}=\sum_{i=0}^{r} \mathfrak{h}_{i},\right.
$$

так как $\left[\mathrm{m}_{i}, \mathrm{~m}_{j}\right]=0$ при $i \neq j$. Покажем, что полученная сумма есть прямая сумма. Пусть $X \in \mathfrak{h}_{i} \cap \mathfrak{h}_{j}$ для некоторого $i \neq j$. Тогда $X \in \mathfrak{g}_{i} \cap \mathfrak{g}_{j}$ и потому

$$
[X, \mathrm{~m}] \subset\left[X, \mathrm{~g}_{0}\right]+\ldots+\left[X, \mathrm{~g}_{r}\right]=0 .
$$

В силу точности линейного представления изотропии $D(\mathfrak{h})$ имеем $X=0, \quad$ т. $\quad$ е. $\quad \mathfrak{h}_{i} \cap \mathfrak{h}_{j}=0 . \quad$ Теперь $\quad \mathfrak{h}=\mathfrak{h}_{0} \oplus \mathfrak{h}_{1} \oplus \ldots \oplus \mathfrak{h}_{r} \quad$ и $\quad \mathfrak{g}=$ $=\mathfrak{g}_{0} \oplus \mathfrak{g}_{1} \oplus \ldots \oplus \mathfrak{g}_{r}$.

Аналогичная теорема для случая римановых естественно-редуктивных пространств была получена Б. Костантом в $\left[{ }^{7}\right]$.

С лед ст ви е. Пусть $\mathrm{G} / \mathrm{H}$ - односвязное естественно-редуктивное однородное пространство с идеально ненулевым умножением в редуктивном дополнении m. Если $G$ проста, то $G / H$ голономно неприводимо относительно естественной связности без кручения.

Док аз а тельство. Простота группы $G$ влечет простоту алгебры m (вследствие результата теоремы 8), что, в свою очередь, указывает нам на неприводимость $G / H$ (теорема 1 ).

Как следует из теоремы 8 , редуктивная пара $(\mathfrak{g}, \mathfrak{h})$ является прямой суммой своих подпар $\left.\left(\mathfrak{g}_{i}, \mathfrak{h}_{i}\right){ }^{10}\right]$ и, в силу соответствия между подпарами редуктивной пары $(\mathfrak{g}, \mathfrak{h})$ и вполне геодезическими подмногообразиями соответствующего редуктивного пространства $G / H\left[{ }^{9}\right]$, имеет место

T еорем а 9. Пусть $G / H-$ односвязное естественно-редуктивное голономно приводимое пространство с разложением $\mathrm{g}=[\mathrm{m}, \mathrm{m}]_{\mathfrak{h}}+\mathrm{m}$ и идеально ненулевым умножениел в $\mathrm{m}$. Тогда имеет место разложение

$$
G / H=G_{0} / H_{0} \times G_{1} / H_{1} \times \ldots \times G_{r} / H_{r},
$$

где каждое $G_{i} / H_{i}$ является вполне геодезическим подмногообразием в $G / H$.

Автор благодарит Ю. Лумисте за внимание к работе.

\section{ЛИТЕРАТУРА}

1. Р ашев ски й П. К. О геометрии однородных пространств. - Тр. семинара по векторному и тензорному анализу, 1952 , вып. 9, с. 49-74.

2. Nomizu, K. Invariant affine connections on homogeneous spaces. - Amer. J. Math., 1954 , v. 76 , p. $33-65$.

3. S a g l e, A. On anticommutative algebras and homogeneous spaces. - J. Math. and Mech., 1967, v. 16, N 12, p. 1381-1393.

4. S a g l e, A. On homogeneous spaces, holonomy and nonassociative algebras. Nagoya Math. J., 1969, v. 32, June, p. 373-394.

5. Ф ляй е р А. Г. Об одном классе редуктивных пространств. - Тр. геом. семинара, 1974 , т. 6 , с. $267-276$. 
6. Kobay a shi, S., N omizu, K. Foundation of differential geometry. New YorkLondon, 1969, v. II, p. 210-316.

7. Kostant, B. On differential geometry and homogeneous spaces II. - Proc. Nat. Acad. Sci., 1956 , v. 42 , p. $354-357$.

8. Kostant, B. On holonomie and homogeneous spaces, - Nagoya Math. J., 1957, v. 12, p. $31-54$.

9. В асильев А. М. О вполне геодезических подмногообразиях однородных пространств. - Докл. АН СССР, 1959, т. 128, № 2, с. $223-226$.

10. Фля йш е р А. Г. Заметки о редуктивных парах. - Уч. зап. Тартуск. гос. ун-та, 1975 , № 355 , c. $27-34$.

Информационно-вычислительный центр Министерства финансов ЭССР

Поступила в редакцию $24 / \mathrm{X} 1977$.

A. FLJAISER

\section{REDUKTIIVSED HOMOGEENSED RUUMID JA MITTEASSOTSIATIIVSED ALGEBRAD}

Olgu $M=G / H$ reduktiivne homogeenne ruum fikseeritud lahutusega $\mathfrak{g}=\mathfrak{h}+\mathfrak{m}$. On teada $\left[{ }^{2}\right]$, et iga $G$-invariantne seostus $\alpha$ ruumil $M$ tekitab mingi mitteassotsiatiivse algebra $(\mathrm{m}, \alpha)$. Artiklis $\left[{ }^{4}\right]$ saadud tulemusi kasutades on käesolevas töös uuritud reduktiivse ruumi $M$ ja temale vastava algebra vastastikuseid seoseid. Põhilist tähelepanu on pööratud reduktiivsetele homogeensetele ruumidele $\left[{ }^{6}\right]$, mille hulgast on vaadeldud nulliteguriteta ideaalidega ruume $\mathrm{m}\left[{ }^{5}\right]$. Niisugused homogeensed ruumid on väga sarnased Riemanni reduktiivsete ruumidega (teoreemid 8,9 ). On märgitud, et kehtib puutuja ruumi $T_{0}(M)$ de Rhami lahutuse analoog.

\section{A. FLAISHER}

\section{REDUCTIVE HOMOGENEOUS SPACES AND NON-ASSOCIATIVE ALGEBRAS}

Let $M=G / H$ be a reductive homogeneous space with a fixed decomposition $\mathfrak{g}=\mathfrak{h}+\mathrm{m}$. It is well known $\left(\left[^{2}\right]\right)$ that for each $G$-invariant connection $\alpha$ on $M$ there exists a nonassociative algebra $(\mathrm{m}, \alpha)$ corresponding to $\alpha$. Using results of $\left(\left[{ }^{4}\right]\right)$, the dependence between the structure of these algebras and the properties of the space $M$ is studied in the present paper. The main part of the paper is devoted to naturally reductive homogeneous spaces $\left(\left[{ }^{6}\right]\right)$. We pick out the class of spaces $G / H$, for which the algebra $\mathrm{m}$ does not contain ideals with zero-multiplication. Those spaces are similar to Riemannian reductive spaces (theorems 8, 9). In particular, the analogue of de Rham's decomposition of the tangent space $T_{0}(M)$ is obtained for them. 